\title{
Energy-Aware Two Link-Disjoint Paths Routing
}

\author{
Gongqi Lin, Sieteng Soh, Mihai Lazarescu \\ Department of Computing \\ Curtin University of Technology \\ Perth, Australia \\ \{gongqi.lin@postgrad.,s.soh@, \\ m.lazarescu@\}curtin.edu.au
}

\author{
Kwan-Wu Chin \\ University of Wollongong \\ Wollongong Australia \\ kwanwu@uow.edu.au
}

\begin{abstract}
Network robustness and throughput can be improved by routing each source-to-terminal $(s, t)$ demand via two linkdisjoint paths (TLDP). However, the use of TLDP incurs higher energy cost. Henceforth, we address the problem of minimizing the energy usage of networks that use TLDP. Specifically, our problem is to maximally switch off redundant network links while maintaining at least $0 \leq T \leq 100 \%$ of $(s, t)$ TLDP in the network, for a given $T$, and limiting the maximum link utilization (MLU) to no greater than a configured threshold. To address this problem, we present a fast heuristic, called TLDP by Shortest Path First (TLDP-SPF), and extensively evaluate its performance on both real and/or synthetic topologies and traffic demands. Our simulation results show that TLDP-SPF can reduce network energy usage, on average, by more than $20 \%$, even for MLU below 50\%. As compared to using Shortest Path routing, while reducing energy by about $20 \%$, TLDP-SPF does not significantly affect $(s, t)$ path length, even for $M L U<50 \%$.
\end{abstract}

Keywords - algorithm; robustness; thoughput; power savings; routing; maximum link utilization; two link-disjoint paths

\section{INTRODUCTION}

Increasing the robustness and reliability of networks are of concern to Internet Service Providers (ISPs), especially given the recent emergence of real time applications such as video on demand and voice over IP [1, 2, 3, 4]. To this end, previous studies $[5,6,7]$ have combined link/node-disjoint paths with QoS routing to guarantee various performance requirements, i.e., reliability and delay. The authors of [5] and [7] consider restorable QoS routing. Specifically, the algorithm in [7] constructs a restoration topology containing a set of bridges, each of which provides backup for a portion of the primary QoS path. In [6], the authors present a problem, called multiple constrained link-disjoint path pair (MCLPP), to find link/nodedisjoint paths in multiple dimensions, and prove its NPCompleteness when one or more link metrics are used. The existence of high path redundancy means there is opportunity to save energy via power-aware traffic engineering [8].

Our paper describes an optimization problem that aims to reduce the energy usage of networks that support two linkdisjoint paths (TLDP). Specifically, our problem aims to reduce network energy usage while ensuring that a network maintains at least $0 \leq T \leq T_{\max }$ percent of all possible $(s, t)$ TLDP and each link's maximum link utilization (MLU) is at most $0 \leq \mathrm{U}_{\mathrm{T}} \leq 1.0$; $T_{\max }$ is the percentage of the total number of $(s, t)$ paths that have at least one TLDP. Specifically, to provide energy efficient, fault-tolerant and high bandwidth routing service to upper layer applications, we route each $(s, t)$ demand through its $(s, t)$ TLDP, if one exists, while powering off idle/unused links to reduce energy expenditure. The MLU constraint is important because ISPs typically bound links' MLU in order to minimize forwarding delay and to absorb any spike in traffic resulting from network failures [10]. In summary, our contributions are twofold: firstly, we pose a problem, called Energy-Aware Two Link-Disjoint Paths Routing (EARTLDP). Our problem is important in reducing the energy usage of networks that employ TLDP to improve fault-tolerance and/or bandwidth/throughput; secondly, we propose a novel algorithm, called Two Link-Disjoint Paths by Shortest Path First (TLDP-SPF), to solve EAR-TLDP. Our algorithm identifies network links that can be powered-off under two constraints: the threshold $T$ and the maximum link utilization $\mathrm{U}_{\mathrm{T}}$. To the best of our knowledge, this is the first algorithm that jointly reduces the number of links in a wired network whilst satisfying these two constraints. We note that reference [8] and [9] have proposed power-aware routing algorithms, but they do not require their generated paths to be link disjoint.

The rest of the paper is organized as follows. Section II defines notations and EAR-TLDP. Section III describes TLDPSPF. Section IV evaluates TLDP-SPF using both real and/or synthetic topologies and data. Finally, Section V concludes the paper.

\section{PROBLEM FORMULATION}

In this section, we first outline all definitions and notations, before formalizing the problem at hand.

\section{A. Notation}

Consider a computer network that is represented by a weighted directed graph $G(V, E)$, where $V$ is the set of $n$ nodes, and $E$ is the set of $m$ links. Each node in $V$ represents a router, and each link $e \in E$ between nodes $v_{i}$ and $v_{j}\left(v_{i}, v_{j} \in V, v_{i} \neq v_{j}\right)$ represents a communication channel with finite capacity/ bandwidth $c(e)>0$. For edge $e$, let $f(e)$ be the total flow on an edge $e$ that has $0 \leq f(e) \leq c(e)$, and hence, its utilization is given by $u(e)=f(e) / c(e)$. This utilization is bound by a given threshold $0 \leq \mathrm{U}_{\mathrm{T}} \leq 1.0$; i.e., any $u(e)$ cannot be larger than $\mathrm{U}_{\mathrm{T}}$. A link's remaining capacity is defined as $r(e)=\mathrm{U}_{\mathrm{T}} * c(e)-f(e) \geq 0$. We assume each link $e$ can be switched-off independently.

Let $D=\left\{d_{i}=\left(s, t, d^{s t}\right) \mid\right.$ demand $i$ from a source node $s=1, \ldots$, $n$ to a terminal node $t=1, \ldots, n$ that has traffic flow $\left.d^{s t}\right\}$. For 
each demand $d_{i}$, let $S P_{i}=\left\{s p_{i q} \mid\right.$ all $(s, t)$ path for $d_{i}$ indexed by $q>0\}$, and $\operatorname{TLD}_{i}=\left\{t l d_{i p} \mid\right.$ all $(s, t)$ TLDP for $d_{i}$ indexed by $\left.p>0\right\}$. Note that $t l d_{i p}=\left\{s p_{i x}, s p_{i y}\right\}$, where $s p_{i x}, s p_{i y} \in S P_{i}$ have no common links. Let $R_{j}$ be a possible set $j$ that contains $|D|$ number of $(s, t)$ single or multi paths with sufficient capacity to route all demands in $D$, i.e., $R_{j}=\left\{R_{j i} \mid\right.$ a set of one or more paths in $S P_{i}$ and/or in $T L D_{i}$ that can be used to route $d_{i}$ \}. The set of all possible solutions to route all demands in $D$ is denoted as $R=\left\{R_{j}|j=1,2, \ldots| R \mid,\right\}$. The length of $R_{j i}, \mathrm{~L}\left(R_{j i}\right)$, is equal to the maximum hop count among all paths in $R_{j i}$ for $d_{i}$, while its bandwidth, $\mathrm{B}\left(R_{j i}\right)$, is calculated from the sum of the smallest $r(e)$ for each path in $R_{j i}$, and by the definition of $R_{j i}$, we have $\mathrm{B}\left(R_{j i}\right) \geq d^{s t}$. We assume the network has sufficient resources, i.e., link capacities, to route all demands. Let $T P_{j} \subseteq R_{j}$ be the set of all $R_{j i} \in R_{j}$ that include at least one $t l d_{i p} \in T L D_{i}$, and $\mathrm{M}\left(R_{j}\right)$ be the percentage of the total number of $(s, t)$ pairs in $R_{j}$ that are routed over TLDPs, i.e., $\mathrm{M}\left(R_{j}\right)=\left|T P_{j}\right| /\left|R_{j}\right| * 100 \%$. Finally, let $\mathrm{S}\left(R_{j}\right)$ be the total number of links used in set $R_{j}$ and $\mathrm{U}\left(R_{j}\right)=\max \left\{f(e) / c(e) \mid \forall e \in R_{j}\right\}$ be the maximum link utilization of $R_{j}$.

\section{B. Problem Statement}

Consider a tuple $\left(G, D, T, \mathrm{U}_{\mathrm{T}}\right)$, where $G(V, E)$ is a network topology, $D$ is a set of traffic demands, $0 \leq T \leq T_{\max }$ is a given threshold, where $0 \leq T_{\max } \leq 100 \%$ is the percentage of the total number of $(s, t)$ pairs that have at least one TLDP, i.e., $T_{\max }=\operatorname{MAX}\left\{\mathrm{M}\left(R_{j}\right)|j=1,2, \ldots| R \mid,\right\}$, and $\mathrm{U}_{\mathrm{T}}$ is the maximum link utilization threshold. Our Energy Aware Two Link-Disjoint Paths Routing (EAR-TLDP) problem is defined as follows.

EAR-TLDP: Find a set of paths $R_{\min } \in R$ that can be used to route all demands in $D$ such that

$$
\begin{aligned}
& \mathrm{S}\left(R_{\min }\right)=\mathrm{MIN}\left\{\mathrm{S}\left(R_{1}\right), \mathrm{S}\left(R_{2}\right), \ldots, \mathrm{S}\left(R_{|R|}\right)\right\}, R_{i} \in R \\
& \mathrm{M}\left(R_{\min }\right) \geq T \\
& \mathrm{U}\left(R_{\min }\right) \leq \mathrm{U}_{\mathrm{T}}
\end{aligned}
$$

Eq. (1) computes the solution, i.e., to find $R_{\min }$ that contains the minimum number of total power-on links. Eq. (2) states that the ratio of the total number of $(s, t)$ pairs that use TLDP for routing in $R_{\min }$ must be no less than a given threshold $T$. Eq. (3) ensures the MLU of links must be no greater than $U_{T}$. Each link $e$ in $R_{\min }$ can be either on or off, and therefore our EARTLDP is a mixed integer-programming (MIP) problem, a wellknown NP-hard problem [11].

\section{GREEN ROUTING ALGORITHMS}

We now present our heuristic called Two Link-Disjoint Paths by Shortest Path First (TLDP-SPF), see Fig. 1, to solve EAR-TLDP. Initially, the set of deleted (remaining) links $E_{d}$ $\left(E_{r}\right)$ is an empty set $(E)$; TLDP-SPF produces $E_{d}$ as its output.

\section{A. TLDP-SPF Algorithm}

As shown in Fig. 1, TLDP-SPF has four main steps, divided into two phases: initialization and re-routing. The initialization phase first generates a set of candidate paths (Step 1), and then uses function TLDP-Routing(), described later, to distribute the traffic of all demands in $D$ through candidate paths (Step 2), and calculate $T_{\max }$. The second phase determines the links that have to be switched off such that remaining links are sufficient to route all traffic demands while satisfying the threshold $0 \leq T \leq T_{\max }$ and MLU constraints.

One can use Yen's algorithm [12] in Step 1 to generate the $k \geq 1$ shortest paths, $K S P_{i}=\left\{s p_{i 1}, s p_{i 2}, \ldots, s p_{i k}\right\}$, for each demand $d_{i}$; we assume each link has a delay of one unit, and thus each $(s, t)$ path length is equal to its $(s, t)$ hop count. Note that for each demand $d_{i}$, we have $K S P_{i} \subseteq S P_{i}$. Let $K S P=\left\{K S P_{i} \mid\right.$ $i=1, \ldots,|D|\}$ be the set of all $k$-shortest paths for all demands in $D$. In Step 2, our algorithm calls function TLDP-Routing(), shown in Fig. 2, that contains two sub-parts to distribute traffic in the network.

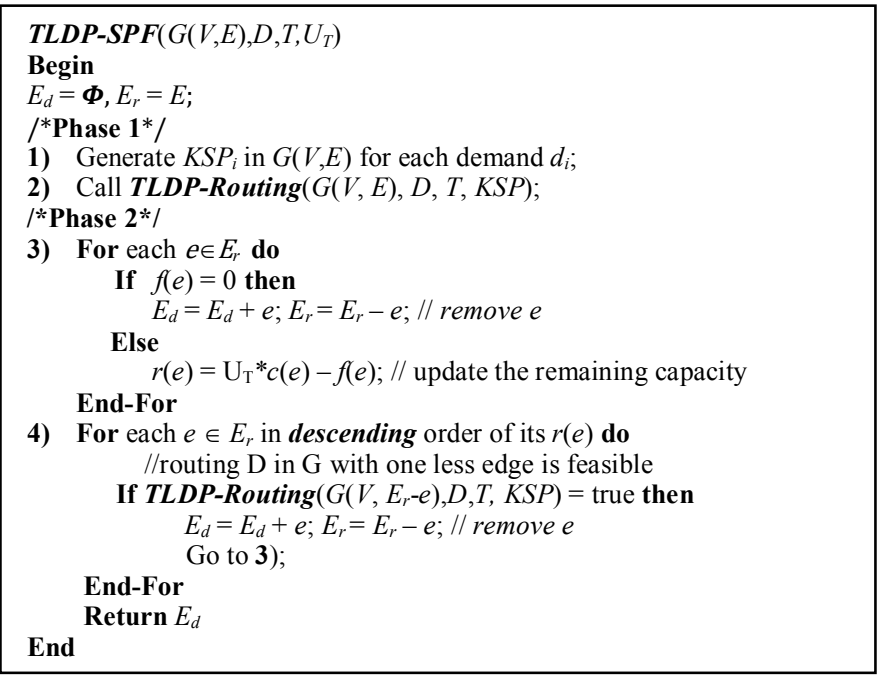

Figure 1. TLDP-SPF algorithm

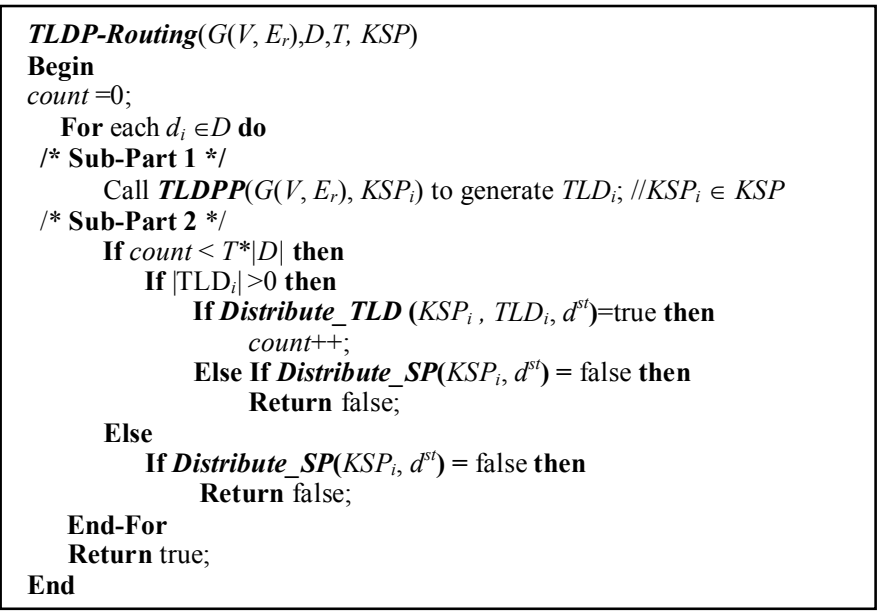

Figure 2. Function TLDP-Routing()

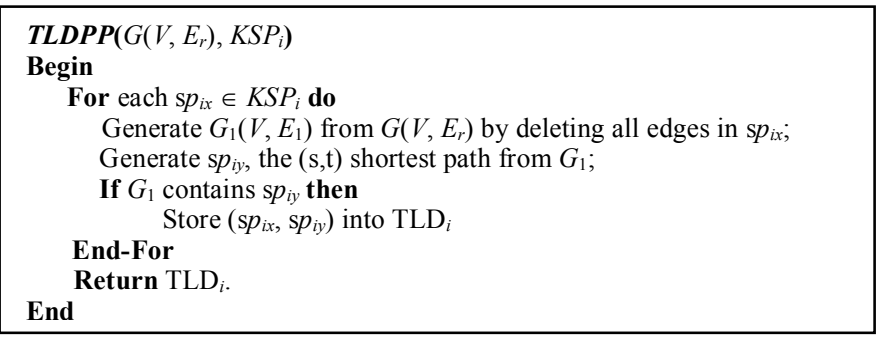

Figure 3. Function TLDPP() 
Sub-Part 1 uses function $\mathbf{T L D P P}()$, shown in Fig. 3, to generate two-link-disjoint paths, $t l d_{i p}$, from each $s p_{i x}$ in $K S P_{i}$. The function stores each $t l d_{i p}$ for $d_{i}$ in set $\mathrm{TLD}_{i}$ in increasing path length order. Sub-Part 2 aims to distribute each traffic demand in $D$ through its TLDP, if possible, i.e., when $\left|\mathrm{TLD}_{i}\right|>0$; otherwise, it calls function Distribute_SP(), shown in Fig. 4, to distribute the traffic volume $d^{x}$ through one or more paths in its $K S P_{i}$.

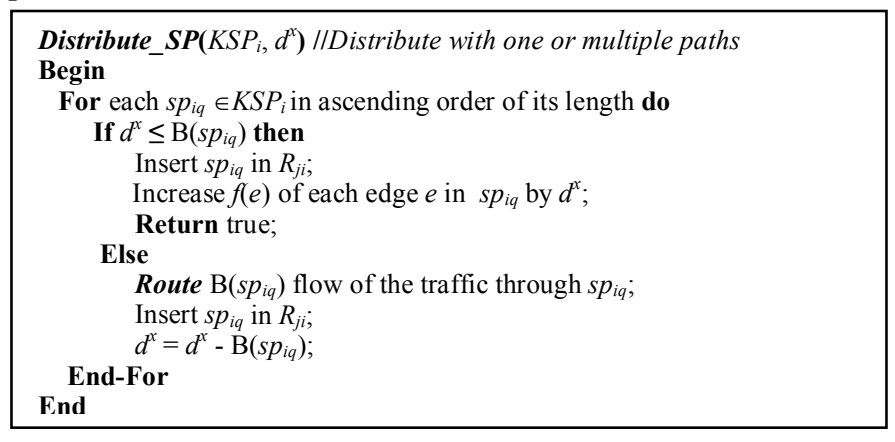

Figure 4. Function Distribute_SP()

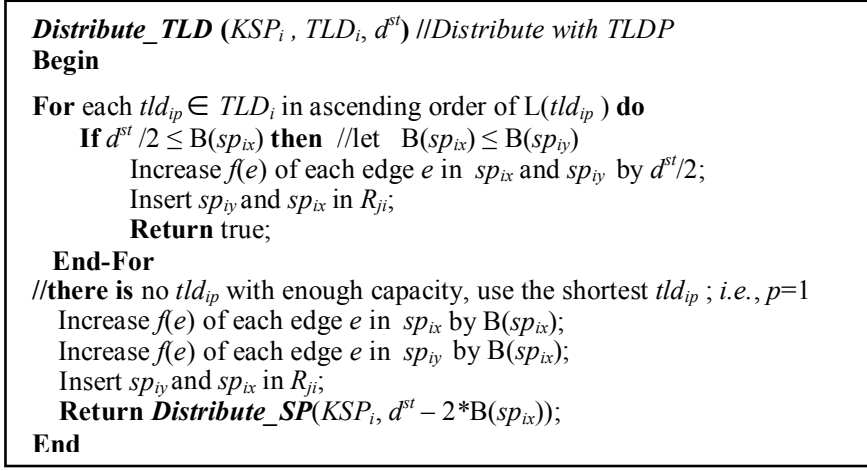

Figure 5. Function Distribute_TLD()

For each demand with $\left|\mathrm{TLD}_{i}\right|>0$, Distribute_TLD () , shown in Fig. 5, routes traffic demand $d^{\text {st }}$ similar to ECMP [13]. That is, it aims to route the traffic of $d_{i}$ evenly through the two paths in $t l d_{i p}=\left\{s p_{i x}, s p_{i y}\right\}$, i.e., with $d^{s t} / 2$ of the traffic in each path and we assume $\mathrm{B}\left(s p_{i x}\right) \leq \mathrm{B}\left(s p_{i y}\right)$. Note, $\mathrm{B}\left(s p_{i q}\right)=\min \left\{r(e) \mid e \in s p_{i q}\right\}$. Specifically, Distribute_TLD() assumes the application requires two link-disjoint paths to carry the same sized traffic since both of them can be a protection path for each other. If $d^{s t} / 2 \leq \mathrm{B}\left(s p_{i x}\right)$, then each path carries traffic size $d^{s t} / 2$, and the selected TLDP is put into $R_{j i}$; otherwise, each path carries traffic of size $\mathrm{B}\left(s p_{i x}\right)$, and the remaining traffic, e.g., $d^{s t}-2^{*}$ $\mathrm{B}\left(s p_{i x}\right)$ is routed through one or more paths in $\mathrm{TLD}_{i}$ and/or $K S P_{i}$. The function updates flow $f(e)$ of each edge $e$ in the selected paths.

Function TLDP-Routing() returns true if it can successfully route all demands in $D$. For this case, it updates only $R_{j i}$ for each affected demand $d_{i}$ and the total flows on each edge $e, f(e)$, used for the demand. Otherwise, it returns false. Notice that, for Phase 1, we assume the network contains sufficient bandwidth to carry the traffic demands, and thus it never returns false. Further, we set $T=1$ so that each demand can be routed through its TLDP whenever possible, and $T_{\max }$, the maximum ratio between the number of demands with $\left|\mathrm{TLD}_{i}\right|>0$ and the total number of demands, as shown in Fig. 2, is set to $T_{\max }=$ count $/|D|$.
Phase 2 uses the initial distribution of traffic and $T_{\max }$ produced by Phase 1 as its inputs, and produces a set of switched-off links and the routes of all demands in $D$. Step 3 switches off each link that is not used to carry any traffic in Phase 1; i.e., each link with $f(e)=0$; otherwise, for all links with $f(e)>0$, it calculates their spare capacity $r(e)=\mathrm{U}_{\mathrm{T}} * c(e)-f(e)$. Step 4 aims to remove a link $e$ with the largest spare capacity since rerouting traffic that goes through the link is more probable. This step uses TLDP-Routing() to check if all traffic can be routed through the remaining edges in $E_{r}$, excluding $e$, while satisfying the constraints that there are at least $T^{*}|D|$ demands that are routed through their TLDP and each link utilization does not exceed $U_{T}$. If the function returns true (feasible), then the step removes $e$ from $E_{r}$, and stores it into $E_{d}$. Note that for this case the function has changed the total flow of each affected edge, and therefore Step 3 is reused to update $E_{r}, E_{d}$ and the remaining capacity of each edge before repeating Step 4 for the next candidate $e$ to be switched off. If TLDPRouting() fails in Step 4, it recovers the status of link $e$, and continue with the remaining edges in $E_{r}$. Thus, Step 4 is iterated no more than $|E|$ times.

\section{B. Time Complexity}

Yen's algorithm [12], used in Step 1 of TLDP-SPF, requires $O(k n(m+n \log n))$ time to generate $k$ shortest paths in a network with $m$ links and $n$ nodes for each demand $d_{i}$; thus Step 1 requires $O\left(\left(|D|^{*} k n(m+n \log n)\right)=O\left(\left(k n^{3}(m+n \log n)\right)\right.\right.$ since $|D| \leq n^{2}$. In Step 2, function TLDPP(), used in TDP-Routing(), requires $O\left(k^{*} n^{2}\right)$ time for each demand $d_{i}$, and thus requires $O\left(n^{2} * k^{*} n^{2}\right)$ for all demands. Further, either function Distribute_TLD() or Distribute_SP () requires, in the worst case, $O\left(k^{*} \bar{m}\right)$ time to route the flow of each $d_{i}$ through its TLD or multiple paths, respectively. Therefore the time complexity of Step 2, i.e., function TLDP-Routing(), is $O\left(k^{*} n^{4}+k^{*} m^{*} n^{2}\right)$. Step 3 is based on the set of edges, so they take $O(m)$ time. Step 4 takes $O(m)$ time to choose a candidate edge and call function TLDPRouting(); therefore the total complexity of Step 4 is $O\left(m^{*}\left(k^{*} n^{4}+k^{*} m^{*} n^{2}\right)\right.$. Lastly, the total complexity of TDP-SPF is $O\left((m+1) *\left(k^{*} n^{4}+k^{*} m^{*} n^{2}\right)+k n^{3}(m+n \log n)\right)=O\left(k^{*} m^{*} n^{4}+\right.$ $\left.k^{*} m^{2} * n^{2}\right)=O\left(k^{*} n^{6}\right)$ since $m=O\left(n^{2}\right)$.

\section{Evaluation}

In this section, we provide experimental findings and present numerical results to confirm the effectiveness of TLDPSPF. We show that it can achieve considerable power savings in real networks with low impact on network performance, such as delay and maximum link utilization.

\section{A. Experiment Setup}

Our experiments are conducted over different network topologies and traffic matrices. We consider three topologies: Abilene [14], GÉANT [15], and the Sprint topology derived from Rocketfuel [16]. We used the Abilene topology and its 288 traffic matrices measured on Sep. $5^{\text {th }}, 2004$ for every 5 minutes within 24 hours - all of which are provided by the authors of [14]. For GÉANT, the 96 traffic matrices [15] used were collected on May 5th, 2005 at an interval of 15 minutes. For Sprint, we set its link capacity following the method in [8], i.e., links connecting Level-1 PoPs are $10 \mathrm{~Gb} / \mathrm{s}$ and the others are $2.5 \mathrm{~Gb} / \mathrm{s}$, and randomly generate a traffic matrix using the gravity model [8]. 
For each network, when using shortest path routing with all links switched-on, Phase 1 of our TLDP-SPF finds only up to $T_{\max }=83.3 \%, 100 \%$, and $37.4 \%$ of the demands in Abilene, GÉANT, and Sprint networks can be routed through their TLDP, respectively. We compute the power saving ratio as the total power of in-active links over the total power of all links in the network. The power consumption of line-cards used in our simulation is specified in [17]. We assume all links use OC3 line cards. Note that as the maximum delay of a single OC-3 link is around $1 \mathrm{~ms}$ [18], the length of $R_{i i}, \mathrm{~L}\left(R_{j i}\right)$, is reported in milliseconds. Our simulations were performed on a Linux PC with $3.07 \mathrm{GHz}$ CPU and $8 \mathrm{~GB}$ RAM. TLDP-SPF requires 3.2, 19.8, and 206.3 CPU seconds, to produce each result for Abilene, GÉANT and Sprint network, respectively.

\section{B. Research Network-Abilene and GÉANT}

Fig. 6(a) shows the power savings (top) and average delay (bottom) for the Abilene network when we set $T=T_{\max }=83.3 \%$, i.e., at its highest constraint, and $\mathrm{U}_{\mathrm{T}}$ from 0.1 to 1.0 . Specifically, for each of the 10 levels of link utilization, we aim to study the effect of reducing energy, i.e., due to switched-off links, on the delay experienced by users while maintaining $T=T_{\max }$. Note that TLDP-SPF could not find any links to be switched off when we set $\mathrm{U}_{\mathrm{T}} \leq 0.2$. For $\mathrm{U}_{\mathrm{T}}=0.3$, TLDP-SPF can switch off between $16.5 \%$ and $20 \%$ of the links except at time $16 \mathrm{~h}$ due to insufficient network capacity during peak periods; see top of Fig. 6(a). Further, for $\mathrm{U}_{\mathrm{T}} \geq 0.4$, TLDP-SPF is able to yield $20 \%$ energy saving while maintaining link utilization to no more than $40 \%$. However, as shown in the bottom of Fig. 6(a), switching off links may affect the average end-to-end delay. While setting $\mathrm{U}_{\mathrm{T}} \geq 0.5$ does not affect delay, reducing $\mathrm{U}_{\mathrm{T}}$ from 0.5 to 0.4 forces TLDP-SPF to find longer alternative paths, and thus increases the average delay by up to $20 \%$.

Fig. 6(b) presents the power saving (top) and average delay (bottom) of GÉANT network when we set $T=T_{\max }=100 \%$. TLDP-SPF is able to switched off $24.67 \%$ of links in the GÉANT network for $\mathrm{U}_{\mathrm{T}}=0.3$ to $\mathrm{U}_{\mathrm{T}}=1.0$, and thus we only show the results for the threshold of MLU no less than 0.3, i.e., $\mathrm{U}_{\mathrm{T}} \geq 0.3$. Notice that $\mathrm{U}_{\mathrm{T}}=0.2$ and $\mathrm{U}_{\mathrm{T}}=0.3$ produce the same energy saving but incur different delays. Although the power saving curve fluctuates during the day due to traffic changes, it always remains around $20.27 \% \sim 25.97 \%$. However, as shown in the bottom of Fig. 6(b), reducing $\mathrm{U}_{\mathrm{T}}$ from 0.2 to 0.1 increases the average delay.

\section{Commercial Network-Sprint}

Given the traffic matrix generated as described in Section IV.A, we scale the matrix using 10 scaling factors to generate 10 different traffic matrices such that when the demands in each matrix are routed using their shortest paths (SP) the MLU in the network is $10 \%$ to $100 \%$, with an increment of $10 \%$. We refer to each traffic matrix as TM_X under SP, where $\mathrm{X}$ is the MLU of the network due to the traffic; e.g., TM_40 is the traffic that produces $\mathrm{MLU}=40 \%$ when using $\mathrm{SP}$ to route demands in TM_40. Finally, for each TM_10 to TM_100, we set $T=T_{\max }=37.4 \%$, and run TLDP-SPF with $\mathrm{U}_{\mathrm{T}}$ from 0.1 to 1.0 .

We found that TLD_SPF produces the same set of routes for the network with TM_10, TM_20 and TM_30 since increasing MLU from $10 \%$ to $30 \%$ only raises most traffics slightly; hence we only report the result for TM_30. We found similar situation for TM_40 to TM_60, and for TM_70 to TM_80. Note that TLD_SPF failed to save energy for TM_90 and TM_100.

Fig. 7(a) shows that the power saving with TM 30 increases from $17.26 \%$ to $19.04 \%$ when $U_{T}$ is set from $0 . \overline{3}$ to 0.5 , and remains at $19.04 \%$ for $\mathrm{U}_{\mathrm{T}}>0.5$. However, the average delay increases to the highest level, i.e., $4.5 \mathrm{~ms}$, when $\mathrm{U}_{\mathrm{T}}=0.4$, decreases when $0.5 \leq \mathrm{U}_{\mathrm{T}} \leq 0.6$, and remains at $4.2 \mathrm{~ms}$ for $\mathrm{U}_{\mathrm{T}}>0.6$. Fig. 7(b) and (c) show the results of TM_50 and TM_70 in terms of power saving and average delay respectively; as shown, they have the same trend as TM 30. However, the latter has a $\mathrm{U}_{\mathrm{T}}$ value, i.e., 0.5 for TM 50 and 0.6 for TM_70 respectively, which means that TLDP_ $\bar{S} P F$ fails to save energy for TM_30 and TM_50 when $\mathrm{U}_{\mathrm{T}}$ is set less than its lower bound on each of the two scenarios. Notice that average delay does not decrease monotonically when $\mathrm{U}_{\mathrm{T}}$ increases since our approach does not consider path delay when switching off links.

\section{The Effects on Link Utilization and Path Delay}

In this subsection, we show the effect of switching off links on link utilization and path delay. We compare results using TDLP-SPF against SP routing as benchmark.

The top of Fig. 8(a) plots the Cumulative Distribution Function (CDF) of link utilization for Abilene. We see that more than $60 \%$ of links when using SP have utilization of at most 0.1 , as compared to at most $30 \%$ in TDLP-SPF; thus SP is better for this case. However, while using only $80 \%$ of total links in Abilene, each switched-on link in TDLP-SPF has utilization of no more than 0.3 , which is better than when using SP, where links may reach a utilization level of 0.5 . We observe a similar trend for GÉANT. As shown in the top of Fig. 8(b), almost $90 \%$ of links in SP has utilization $u_{i j} \leq 0.05$, better than only $53.68 \%$ in TLDP-SPF. However, while switching off $22.97 \%$ of total links in the network, 98.65\% links in TLDP-SPF have $u_{i j} \leq 0.1$, better than $93.64 \%$ of all links for SP. The results for Abilene and GÉANT show that TDLP-SPF is able to distribute traffic flows more evenly than SP routing. The bottom of Fig. 8(a) and 8(b) plot the CDF of the path delay for Abilene and GÉANT, respectively. Both figures show shorter hop counts when using SP. This is expected because SP routes each traffic through its shortest path and hence is optimal in terms of path delay. Further, SP routes all traffic demands through more switched-on links, providing more options on shortest paths, than TLDP-SPF.

\section{E. The Effects of Threshold T on Energy Savings}

As shown in Fig. 9(a), the average energy saving on the Abilene network is $46.38 \%$, when $T$ is set to $0 \%$, but reduces sharply to around $20 \%$ when $T \geq 13.3 \%$. Similarly, Fig. $9(\mathrm{~b})$ and Fig. 9(c) show the average energy saving on GEANT and Sprint decreases by more than half, from $52.7 \%$ to $23.26 \%$ and from $39.29 \%$ to $19.05 \%$ when $T$ increases from $0 \%$ to $100 \%$ and from $0 \%$ to $37.4 \%$, respectively. Notice that for all three networks, after a sharp decline in energy saving at the following $T$ values, $T=13.3 \%, T=15 \%$, and $T=8 \%$ for Abilene, GÉANT, and Sprint respectively, increasing $T$ does not significantly affect their respective energy saving. Thus, networks that support TLDP should use TLDP-SPF with the highest $T$ possible, i.e., set $T=T_{\max }$ to optimize the faulttolerance and performance of applications. 


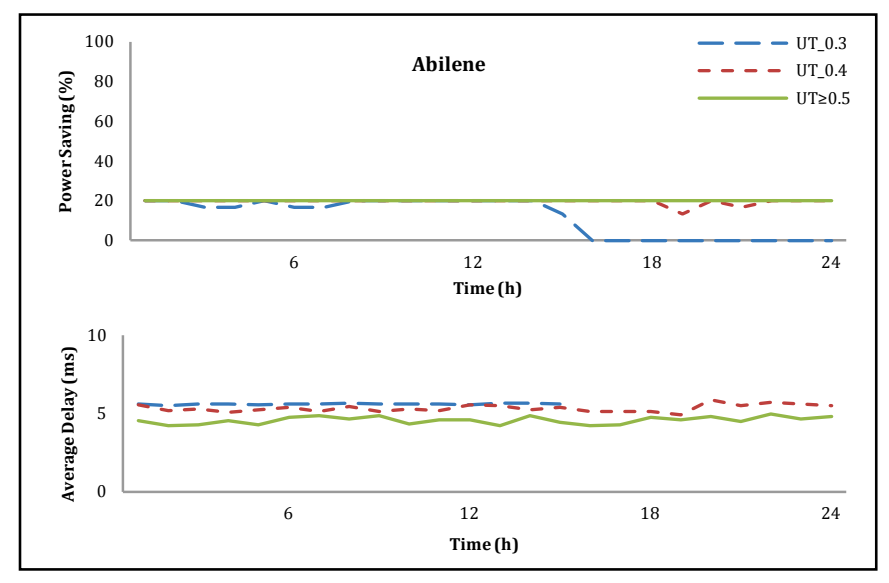

(a) Abilene ( $\left.T=T_{\max }=83.3 \%\right)$

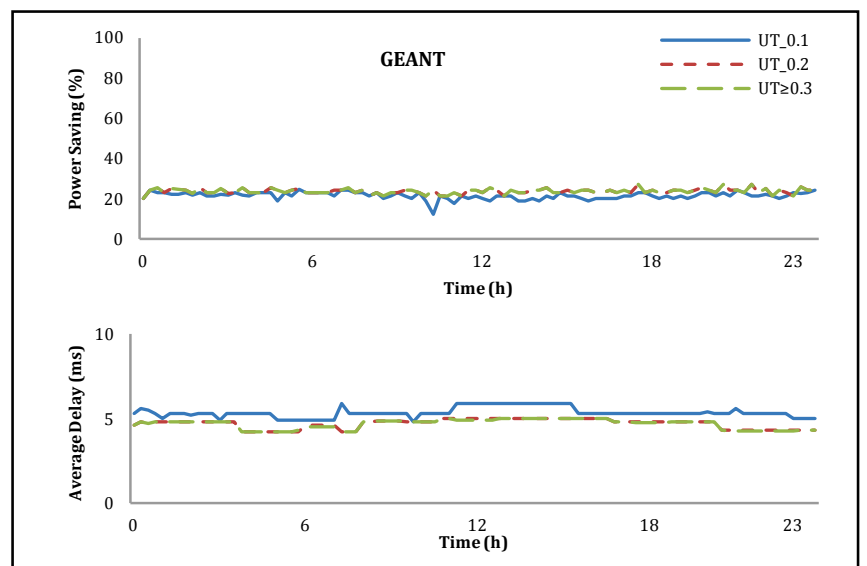

(b) GÉANT $\left(T=T_{m a x}=100 \%\right)$

Figure 6. Energy saving and average delay on Abilene and GÉANT

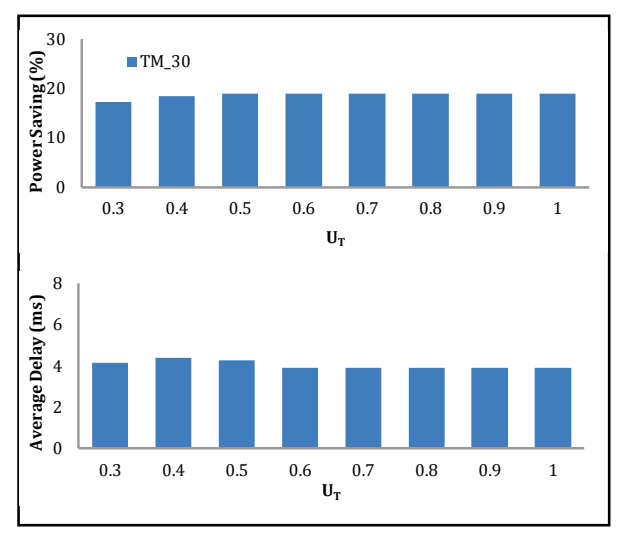

(a)TM_30

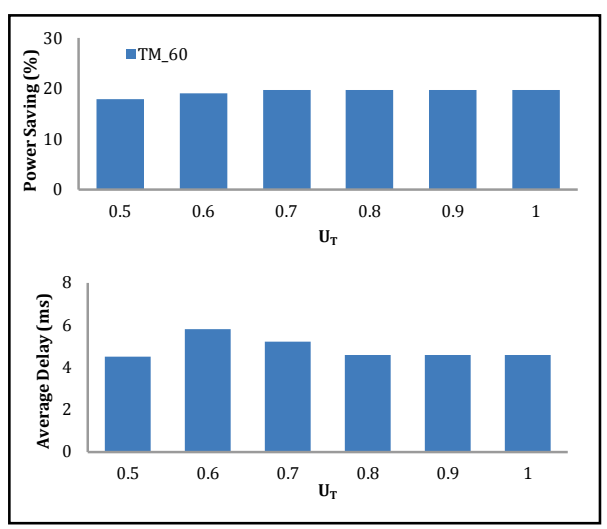

(b)TM_60

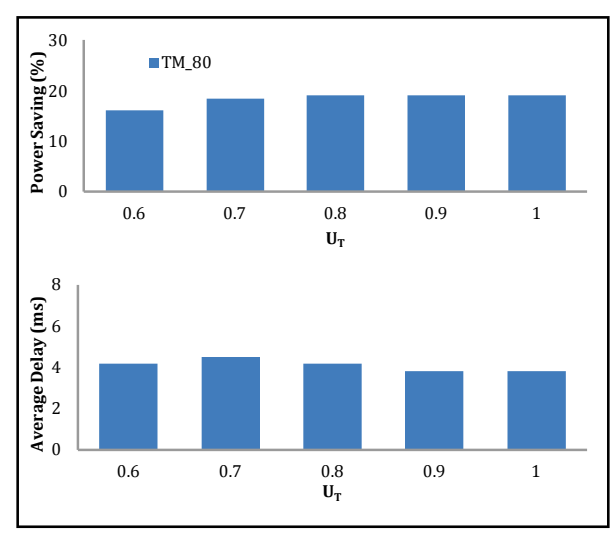

(c) TM_80

Figure 7. Energy saving and average delay on Sprint with different MLUs under SP

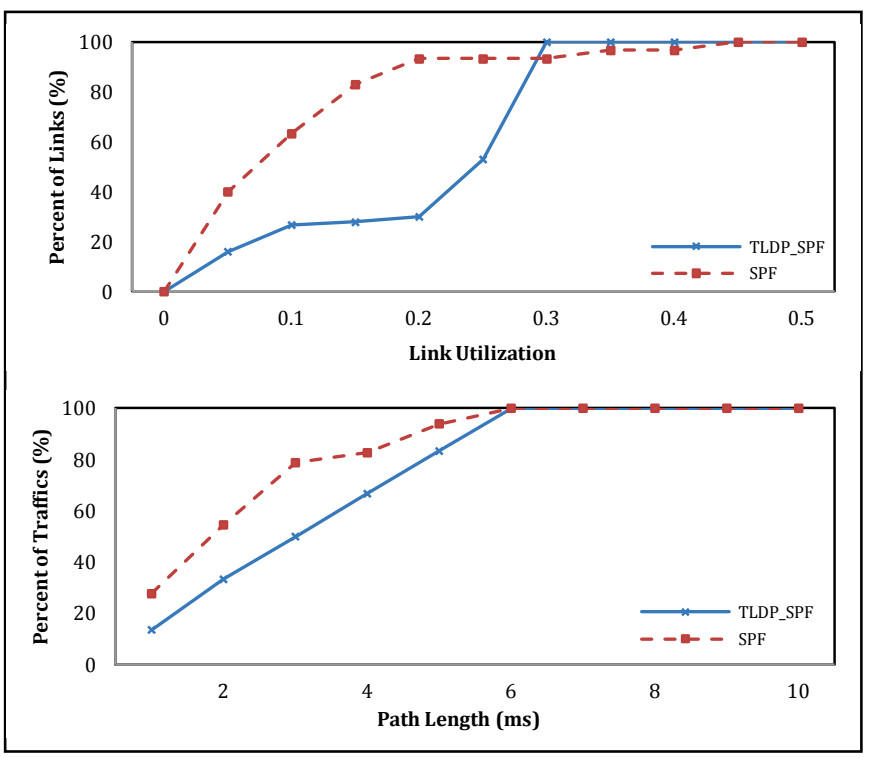

(a) Abilene ( $T=T_{\max }=83.3 \%$ and $\left.\mathrm{U}_{\mathrm{T}} \geq 0.5\right)$
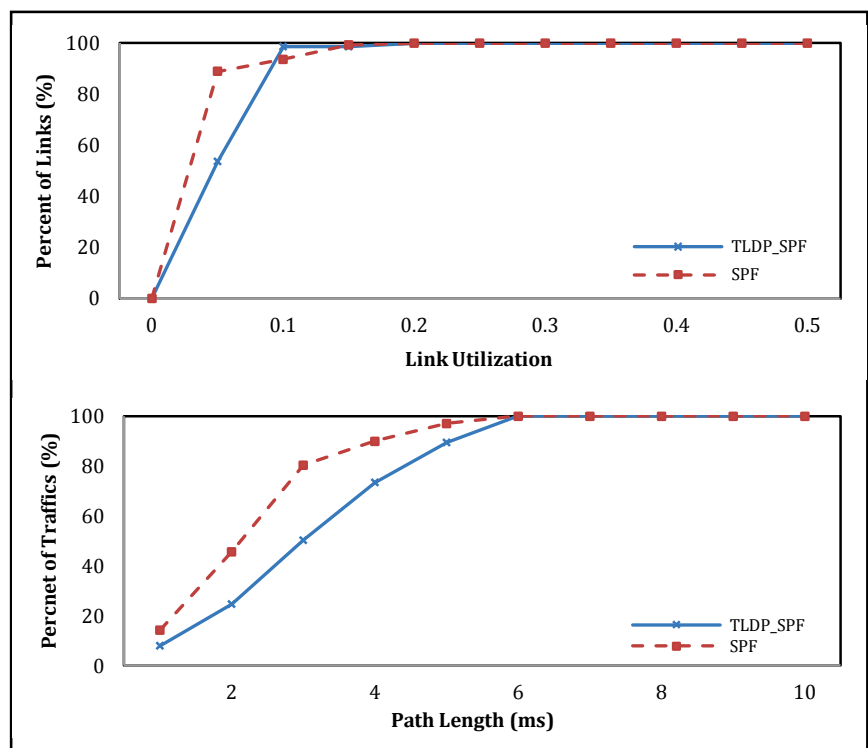

(b) GÉANT ( $T=T_{\max }=100 \%$ and $\left.\mathrm{U}_{\mathrm{T}} \geq 0.3\right)$

Figure 8. CDF of link utilization and path delay on Abilene and GÉANT 


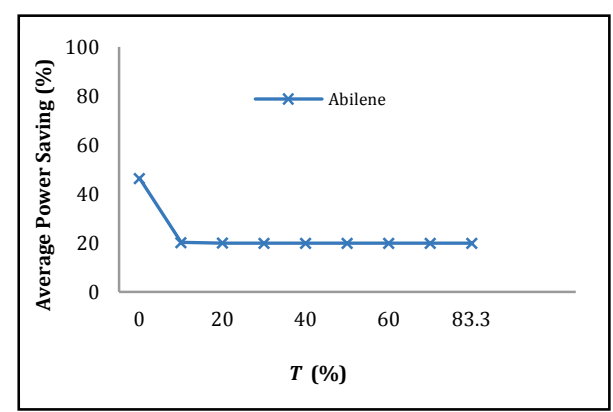

(a)Abilene

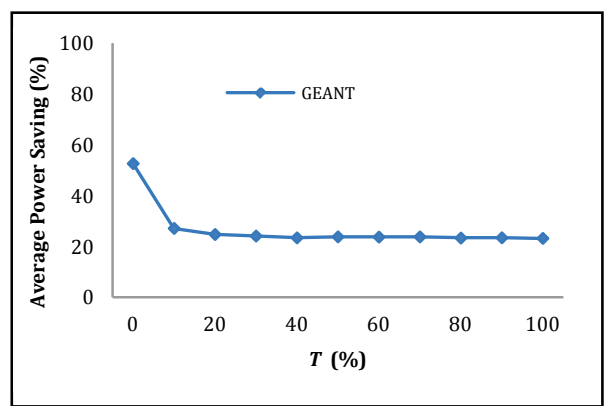

(b) GÉANT

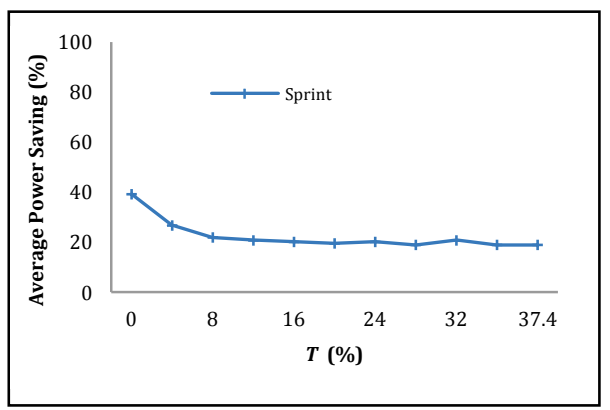

(c) Sprint

Figure 9. Energy saving for different thresholds of $T$

\section{CONCLUSION}

We have presented a new energy-aware routing problem. To reduce energy expenditure, we aim to maximally switch off unnecessary links during off-peak periods such that the remaining powered on links are sufficient to route the given traffic demands under the constraints that the ratio of using TLDP is not less than a given threshold $T$ and the maximum link utilization is not greater than a threshold $\mathrm{U}_{\mathrm{T}}$. We have proposed an efficient and effective heuristic technique to solve the problem. Through extensive simulations on both real and synthetic network topologies and traffic demands, we have shown its benefits in reducing a network's energy consumption.

\section{REFERENCES}

[1] K. Xiong, Z. D. Qiu, Y. Guo, and H. Zhang. "Multi-constrained shortest disjoint paths for reliable QoS routing. " ETRI Journal, 31(5): 534-544, 2009.

[2] G. Apostopoulos, R. Guerin, S. Kamat, and S.K. Tripathi. "Quality of service based routing: A performance perspective." In ACM SIGCOMM 1998, pp. 17-28.

[3] J. Chen, R. Sundaram, M. Marathe, and R. Rajaraman. "The confluent capacity of the Internet: congestion vs. dilation." In IEEE ICDCS 2006.

[4] W. Zhang, J. Tang, C. Wang, and S. D. Soysa. "Reliable adaptive multipath provisiong with bandwidth and differential delay constraints." In IEEE INFOCOM 2010.

[5] M. Kodialam, T. V. Lakshman, "Restorable dynamic quality of service routing”. In IEEE Communications Magazine 2002;72-81.

[6] Y. Guo, F. Kuipers and P. V. Meeghem. "Link-disjoint paths for reliable QoS routing.” In Int. J. Commun. Syst., 26:779-798, 2003.
[7] Y. Bejerano, Y. Breitbart, A. Orda, R. Rastogi and A. Sprintson, "Algorithms for computing QoS paths with restoration". In IEEE INFOCOM 2003.

[8] M. Zhang, C. Yi, B. Liu, and B. Zhang, "GreenTE: Power-Aware Traffic Engineering". In ICNP, 2010.

[9] W. Fisher, M. Suchara, and J. Rexford, "Greening backbone networks: reducing energy consumption by shutting off cables in bundled links". In Green Networking, 2010.

[10] M. Kodialam, T. V. Lakshman, J. B. Orlin and S. Sengupta, " Preconfiguring IP-over-optical networks to handle router failures and unpredictable traffic". In IEEE INFOCOM 2006.

[11] T. H. Cormen, C. E. Leiserson, R. L. Rivest, C. Stein, "Introduction to algorithms (Second Edition)". MIT Press, 2005.

[12] J. Y. Yen, "Finding the K Shortest Loopless paths in a network". In Management Science, 17(11), 1971.

[13] Multipath Issues in Unicast and Multicast Next-Hop Selection, available at http://www.ietf.org/rfc/rfc2991.txt.

[14] "Yin Zhang's Abilene TM". http://www.cs.utexas.edu/ yzhang/ research/ AbileneTM/.

[15] S. Uhlig, B. Quoitin, J. Lepropre, and S. Balon, "Providing Public Intradomain Traffic Matrices to the Research Community". ACM SIGCOMM Computer Communication Review, Vol. 36, no. 1, pp. 8386, January 2006.

[16] N. Spring, R. Mahajan, D. Wetherall, and T. Anderson, "Measuring ISP Topologies with Rocketfuel" In IEEE/ACM Transactions on Networking, vol. 12, no. 1, pp.2-16, 2004.

[17] "Power Management for the Cisco 12000 Series Router." [Online]. Available:http://www.cisco.com/en/US/docs/ios/12_0s/feature/guide/12s power.html.

[18] K. Papagiannaki, S. Moon, C. Fraleigh, P. Thiran and C. Diot, "Measurement and Analysis of Single-Hop Delay on an IP Backbone Network". In IEEE Journal on Selected Areas on Communications, vol. 21, No. 6, Aug 2003. 